\title{
Finite Element Mesh Considerations for Reduced Integration Elements
}

\author{
Gordon S. Bjorkman ${ }^{1}$ and Jason M. Piotter ${ }^{1}$
}

1) Spent Fuel Storage and Transportation Division, U.S. Nuclear Regulatory Commission

\begin{abstract}
Finite element models of spent fuel casks and canisters that are typically used in impact and impulse analyses may contain tens of thousands of nonlinear elements. These models use explicit time integration methods with small time steps, and therefore, to achieve reasonable run times, fully integrated elements are replaced with under-integrated elements that use reduced integration procedures. When fully integrated these elements produce a linear strain distribution. Reduced integration, however, results in a constant strain distribution, which requires more elements through the thickness of the canister shell to achieve the same accuracy as fully integrated elements. This paper studies the effect of the number of reduced integration elements through the thickness of the shell of a canister and the element height to shell thickness ratio on the accuracy of the strains in regions of high through thickness bending, such as the junction between the shell and base plate. It is concluded that mesh refinement has a significant effect on the maximum plastic strain response in such regions and that a converged solution may not be attainable within practical limits of mesh refinement. Therefore, guidance for finite element meshing needs to be developed from benchmark testing and analysis if strain based acceptance criteria are to be used.
\end{abstract}

\section{INTRODUCTION}

Impact and impulse analyses often lead to the use of explicit time integration methods because of their efficiency when dealing with the small time steps needed to resolve high frequency shock waves. For large models, typically containing tens of thousands of nonlinear elements, computer run times may be excessive. Therefore, to achieve reasonable run times fully integrated elements are replaced with under-integrated elements that use reduced integration procedures. For three dimensional models, run-time reductions of an order of magnitude are often achieved using reduced integration instead of full integration.

Drop impact analyses of spent fuel transportation casks that are conducted to satisfy the requirements of 10 CFR 71.73 typically have shell and lid components constructed of 8-node brick elements. When fully integrated, i.e., 2x2x2 Gauss quadrature, these elements produce a linear strain distribution. Reduced integration, however, results in a constant strain distribution, which requires more elements through the thickness of the canister shell to achieve the same accuracy as fully integrated elements. This paper studies the effect of the number of reduced integration elements through the thickness of the cylindrical shell and the element height to shell thickness ratio on the accuracy of the strains in regions of high through thickness bending, such as the junction between the shell and lid or shell and base plate. The loading case considered is an internal pressure pulse. A follow-up paper will address drop impact.

\section{METHODOLOGY}

To evaluate the performance of reduced integration constant stress 8 node solid elements, a finite element cylindrical shell model was constructed. The $1.27 \mathrm{~cm}$ (0.5 inch) cylindrical shell is 305 
cm (120 inches) long and $173 \mathrm{~cm}$ (68 inches) in diameter and is welded to a $15 \mathrm{~cm}$ (6.0 inch) thick base plate. The number of solid elements considered through the shell thickness is 2, 3 and 5 . To determine the effect of the length of the elements on response, the longitudinal height of the elements varies from a height to thickness ratio of 0.156 to 5.0. A sketch of the finite element model at the intersection of the shell and base plate in Figure 1 shows the element height (h), shell radius (R) and shell thickness (t) for the case of 5 elements through the thickness. It is this first layer of shell elements adjacent to the base plate where response is evaluated, since this is the location of the weld between a typical spent fuel canister shell and base plate, as well as, the location of the highest plastic strains under drop impact or internal pressure pulse.

The internal pressure loading in the analysis is applied to the shell only and consists of a pressure pulse with a linear rise time of 5.0 msec to a constant pressure of $5.52 \mathrm{MPa}$ (800 psi). The shell and base plate material is stainless steel with a stress strain curve of the form, $\sigma=K \varepsilon^{n}$, where, $\mathrm{K}$, is $1104 \mathrm{MPa}(160,000 \mathrm{psi})$ and, $\mathrm{n}$, is 0.279 . The LS-DYNA computer code [1] was used for this study.

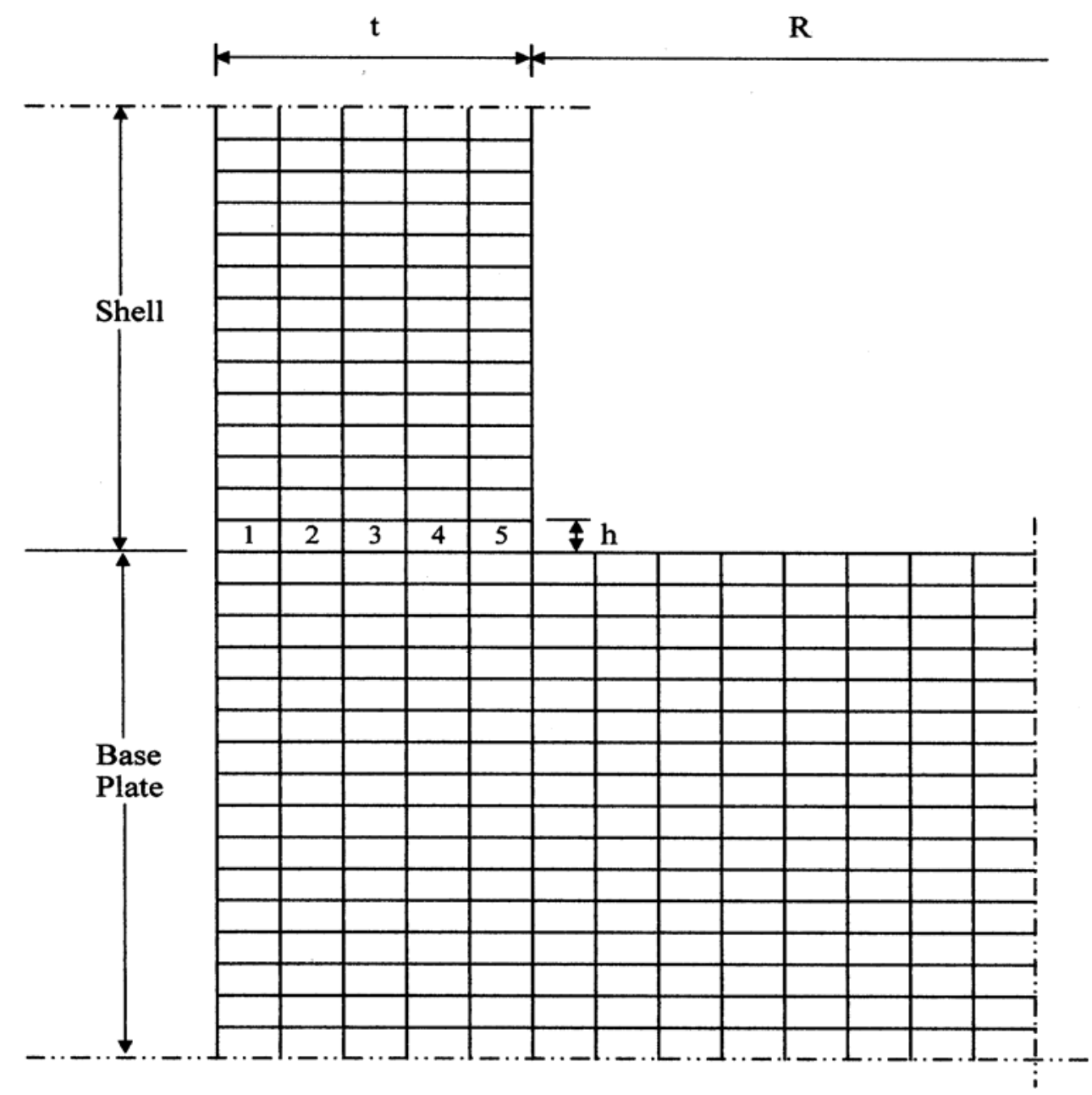

Figure 1: Sketch of the finite element model at the intersection of the shell and base plate showing the element height $(h)$, shell radius $(R)$ and shell thickness (t) for the case of 5 elements through the thickness. 


\section{RESULTS}

Since acceptance criteria based on strain is the best measure of containment boundary integrity, effective plastic strain in each element through the thickness is tabulated at the time when maximum response is achieved. Figure 2 plots the maximum effective plastic strain for 5 elements through the thickness against the element height to shell thickness ratio. It is clear from this Figure that the element height to thickness ratio at the shell to base plate junction has a dramatic effect on the maximum strain response. Figure 3 is the same as Figure 2, but also includes the results for 2 and 3 elements through the thickness. These results show that mesh refinement, both in the number of elements through the thickness and the relative height of the elements, has a significant influence on maximum strain response. The results also show that a converged solution was not obtained even for the finest mesh used in this study.

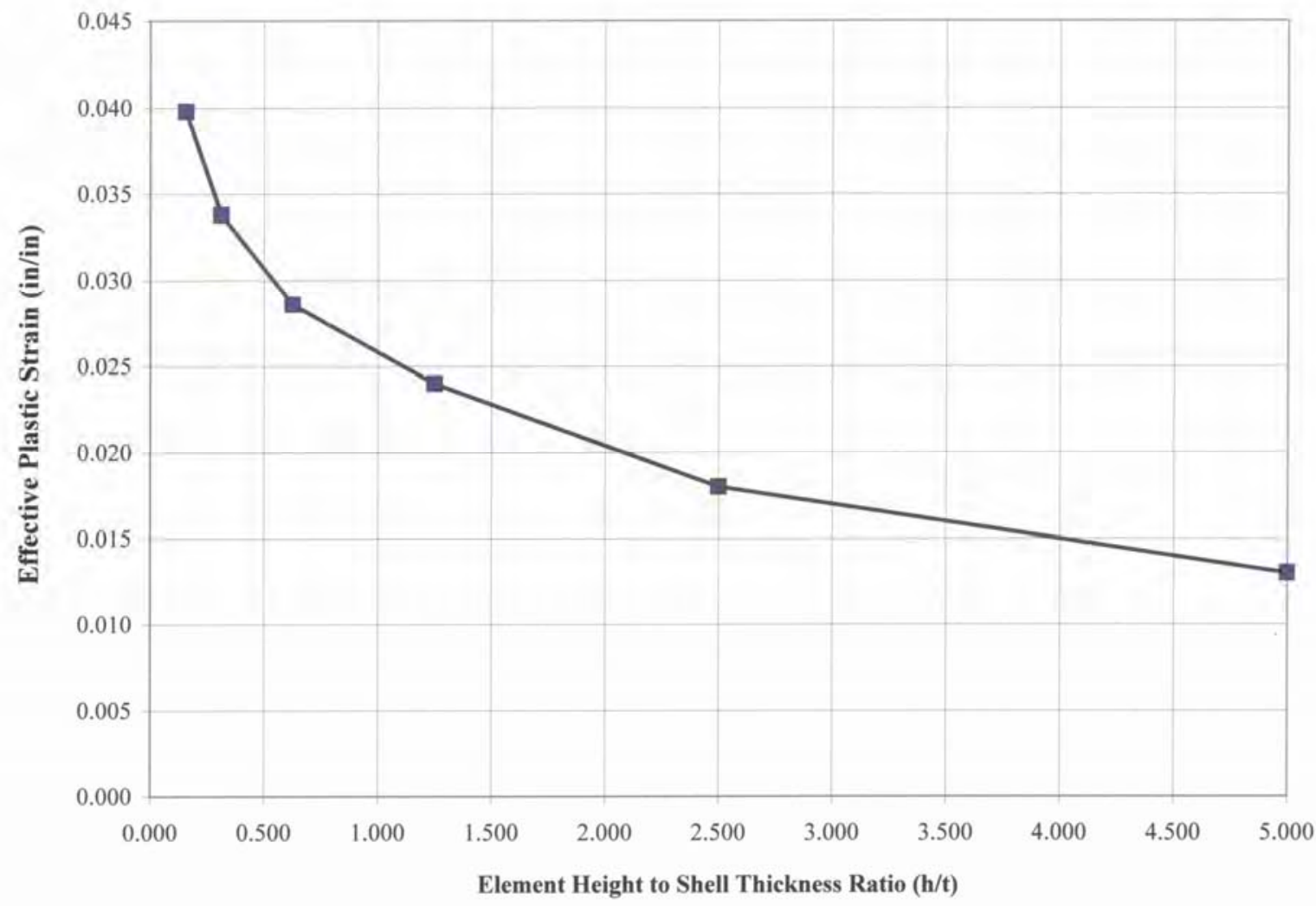

Figure 2: Maximum effective plastic strain for 5 elements through the thickness plotted against the element height to shell thickness ratio. 


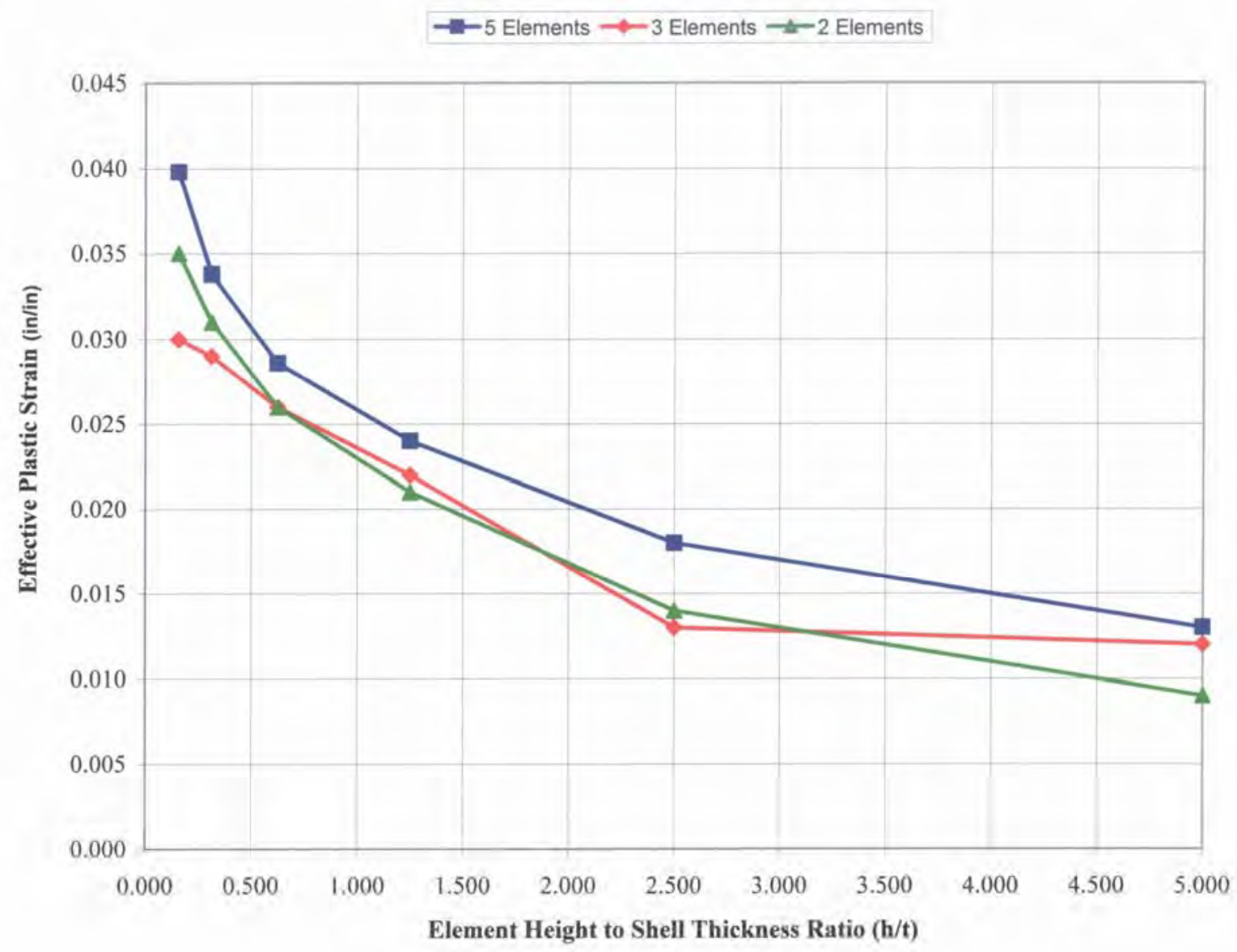

Figure 3: Maximum effective plastic strain for 2, 3 and 5 elements through the thickness plotted against the element height to shell thickness ratio.

\section{CONCLUSION}

The use of reduced integration elements requires special attention to mesh refinement in regions of high strain gradient, such as at structural discontinuities. It has been shown that mesh refinement has a significant effect on the maximum plastic strain response in such regions, and that a converged solution may not be attainable within practical limits of mesh refinement. Therefore, guidance for finite element meshing needs to be developed from benchmark testing and analysis if strain based acceptance criteria are to be used.

\section{REFERENCES}

1. Livermore Software Technology Corporation, LS-DYNA Computer Code, Version 970. 